Meta

Journal des traducteurs

Translators' Journal

\title{
La Banque de terminologie au service de l'entreprise
}

\section{Bruno Couture}

Volume 21, numéro 1, mars 1976

La traduction et l'entreprise

URI : https://id.erudit.org/iderudit/002429ar

DOI : https://doi.org/10.7202/002429ar

Aller au sommaire du numéro

Éditeur(s)

Les Presses de l'Université de Montréal

ISSN

0026-0452 (imprimé)

1492-1421 (numérique)

Découvrir la revue

Citer cet article

Couture, B. (1976). La Banque de terminologie au service de l'entreprise. Meta, 21(1), 100-109. https://doi.org/10.7202/002429ar d'utilisation que vous pouvez consulter en ligne.

https://apropos.erudit.org/fr/usagers/politique-dutilisation/ 


\section{La Banque de terminologie au service de l'entreprise*}

Les participants à la vi ${ }^{\mathrm{e}}$ Biennale de la langue française qui s'est tenue l'automne dernier à Echternach (Luxembourg) ont pu assister le 10 septembre au déroulement de l'Opération Mercure dont le succès a été rendu possible grâce au concours empressé de la Banque de terminologie de la C.C.E. (Commission des Communautés européennes). Il s'agissait de la première consultation intercontinentale d'un dictionnaire multilingue automatique, celui de la Banque de terminologie de l'Université de Montréal ${ }^{1}$. Comme cet événement survenait juste après une fructueuse expérience de liaison permanente entre cette banque et un bureau de traduction canadien du secteur privé, il a fourni aux deux initiateurs du projet, M. René Deschamps, directeur des Services linguistiques de la société Bell Canada, et M. Marcel Paré, directeur de la Banque de terminologie de l'Université de Montréal ${ }^{2}$, une tribune appropriée pour livrer publiquement, dans des communications jumelées, les premiers résultats de leur collaboration. Or, même si le texte de leurs exposés figurera vraisemblablement dans les Actes de la biennale, il est apparu à la direction de Meta qu'un numéro spécial sur la traduction dans l'entreprise serait incomplet sans un compte rendu de cette heureuse innovation.

\section{CHOIX DU CLIENT}

Avant de décrire l'expérience proprement dite, il importe de souligner que les qualités et les dispositions de l'interlocuteur choisi comme partenaire par la Banque de terminologie ne sont pas étrangères à cette réussite. En effet, il fallait un client qui réponde à certains critères quantitatifs et qualitatifs. Du point de vue de la quantité, c'est la taille du groupe à desservir qui entrait en ligne de compte ; on doit entendre par là que, pour une première expérience, l'abonné idéal est un service de traduction d'effectif moyen. La raison en est simple : un bureau au personnel très nombreux risquait de soumettre la Banque à une demande trop forte pour son actuelle capacité de réponse au service d'appoint (S.V.P.). De même, un bureau au personnel trop restreint aurait fourni des résultats statistiquement peu significatifs et donc difficilement extrapolables pour une clientèle plus populeuse.

* Cf. Robert Dubuc, "Définition d'une démarche terminologique en fonction des besoins à satisfaire ", Meta, vol. 18, $\mathrm{n}^{\text {os }}$ 1-2, mars-juin 1973, p. 269-276.

1. Cf. Robert Dubuc, "La Banque de terminologie de l'Université de Montréal », la Banque des mots, $\mathrm{n}^{\circ} 3,1972$, p. 35 à 50 .

2. Cf. Marcel Paré, "Pourquoi une banque au lieu d'un dictionnaire ", Meta, vol. 17, $\mathrm{n}^{\circ} 3$, septembre 1972, p. 152 à 159. 
Sur le plan qualitatif, deux critères étaient à retenir: la compétence et la motivation. Dans l'optique de la Banque, le client choisi devait, pour offrir la compétence professionnelle nécessaire, avoir un service de linguistique établi depuis un certain nombre d'années, qui posséderait par conséquent une bonne expérience de la traduction, mais il était également souhaitable qu'il dispose déjà d'au moins un ou deux terminologues. Ainsi seraient évitées, estimait-on, les avalanches de demandes S.V.P. que n'aurait pas manqué de susciter, par exemple, le choix d'un service de traduction en voie d'organisation, et aussi les problèmes divers qui auraient sans doute découlé d'une méconnaissance des aspects pratiques de la recherche terminologique. Quant au critère de la motivation, les promoteurs du projet, aux Services linguistiques et à la direction de Bell Canada, offraient sous ce rapport toutes les garanties souhaitables, notamment en raison du fait que la société exerce son activité dans le secteur des télécommunications, qui sont des auxiliaires indispensables de la téléinformatique. On conçoit done facilement que, dans ces conditions, l'expérience s'est engagée sous de très heureux auspices.

\section{LES OBJECTIFS}

Du point de vue de la Banque, l'expérience visait deux objectifs principaux. Elle était destinée premièrement à recueillir le plus grand nombre possible de données statistiques, soit pour qu'on en tire des leçons pratiques sur le plan de l'organisation et du fonctionnement, soit pour qu'on puisse apporter des réponses sûres aux demandes de renseignements des clients en puissance. En second lieu, l'opération devait permettre de connaître, au moyen d'un sondage approprié, les réactions des utilisateurs vis-à-vis la consultation, le dictionnaire automatique et le service d'appoint (S.V.P.).

\section{DURÉE DE L'EXPÉRIENCE}

La durée de l'expérience avait été fixée initialement à six mois. Il faut bien reconnâtre que ce choix n'est pas tout à fait arbitraire, puisqu'il facilite à priori les extrapolations susceptibles de fournir des ordres de grandeur pour une année entière d'exploitation. On admettra néanmoins que la plus extrême prudence est de rigueur en cette matière, les conditions d'une expérience étant souvent, par définition, très éloignées de la réalité.

Malheureusement, le rodage a été marqué par des pannes mécaniques d'une durée et d'une fréquence exceptionnelles, les unes consécutives à la mise en place d'un nouvel ordinateur, les autres attribuables à une surcharge temporaire de l'équipement informatique. C'est ainsi que, pendant trois ou quatre semaines, le service sporadique a rendu presque impossible l'interrogation du fichier automatique. Ce fâcheux contretemps devait même amener les parties à s'entendre pour prolonger l'expérience de quelques mois, notamment à titre de compensation pour l'abonné. En ce qui concerne les statistiques, il va de soi que la prise en compte de ces semaines creuses dans les calculs risquerait de fausser dangereusement les résultats. Aussi allons-nous présenter ci-après comme semestrielles des données qui, en fait, ont été recueillies sur une période brute de sept mois, allant du 11 février au 11 septembre 1975 . 


\section{LE FICHIER TERMIUM}

En vertu du contrat d'abonnement, l'usager avait droit à l'interrogation directe du fichier automatique Termium ${ }^{3}$ et au service d'appoint qui en constitue à l'heure actuelle le complément indispensable, en raison du volume encore relativement faible du capital en mémoire. À titre indicatif, mentionnons qu'en juillet 1975, la composition de ce capital par type de fiche était la suivante : 47711 fiches avec contextes anglais et français $(64 \%), 2802$ fiches avec contexte anglais seulement (4\%), 4249 fiches avec contexte français seulement (6\%), 19291 fiches sans aucun contexte $(26 \%)$, soit un total de 74053 fiches. D'autre part, la ventilation du fichier par domaines d'emploi s'établissait approximativement ainsi :

$\begin{array}{cc}\text { REPARTITION APPROXIMATIVE DU CAPITAL TERMINOLOGIQUE DE LA } \\ \text { PAR DOMAINES D'EMPLOI, AU J JUILLET 1975 } \\ \text { (Classement par ordre de grandeur décroissant) } \\ \begin{array}{c}\text { Domaines } \\ \text { Administration et gestion }\end{array} \\ \text { Télécommunications et postes } & 12 \\ \text { Industries } & 10 \\ \text { Économie } & 9 \\ \text { Sciences humaines } & 7 \\ \text { Électricité } & 7 \\ \text { Sciences mathématiques, physiques et naturelles } & 6,5 \\ \text { Construction } & 6,5 \\ \text { Sciences du milieu } & 6 \\ \text { Arts, loisirs, sports } & 6 \\ \text { Transport et manutention } & 5 \\ \text { Cybernétique } & 5 \\ \text { Médecine } & 3,5 \\ \text { Agriculture } & 3,5 \\ \text { Droit } & 2,5 \\ \text { Mécanique et chaleur } & 2,5 \\ \text { Métallurgie } & 2 \\ \text { Chimie } & 2 \\ \text { Économie domestique et hôtellerie } & 1,5 \\ \text { Mines et carrières } & 1 \\ \text { Pétrole } & 0,5 \\ \text { Défense nationale } & 0,5 \\ & 0,5\end{array}$

À ce propos, il semble opportun de s'arrêter un moment sur les sources d'alimentation du fichier Termium, car au Canada deux courants d'opinion divisent actuellement les consommateurs de terminologie sur cette importante question. En effet, la majorité des traducteurs qui comptent un certain nombre d'années d'exercice, s'estimant aptes à faire eux-mêmes un choix judicieux parmi les innombrables solutions que pourrait offrir la machine, préconisent un stockage massif de tous les travaux terminologiques existants, sans égard à leur qualité, quitte à faire traiter la matière une fois l'emmagasinage terminé ; tandis qu'une minorité, qui se recrute entre autres chez les jeunes diplômés des écoles de traduction où la terminologie est une matière d'enseignement à part entière, prône la sélection à l'entrée. La question est complexe et mérite une études approfondie, qui sortirait évidemment du cadre de cet article. On nous permettra néanmoins de faire observer que la

3. Cf. Robert Dubuc, "Termium System Description ", Meta, vol. 17, n 4, décembre 1972, p. 203 à 219. 
prétention des praticiens d'expérience à pouvoir opérer un choix éclairé parmi des données terminologiques non traitées au préalable, n'est fondée que dans la mesure où il y aurait toujours une étroite concordance entre leurs champs de spécialisation et les sujets abordés dans les textes qu'ils doivent traduire, ce qui n'est pas le cas actuellement.

Quoi qu'il en soit, la Banque de terminologie de l'Université de Montréal, pour sa part, tend à respecter les critères de qualité de l'information terminologique qu'elle a adoptés à l'origine. Ce qui ne l'empêche pas d'emmagasiner parfois, faute de mieux, mais à titre provisoire, des données auxquelles elle doit attribuer des cotes de fiabilité de deuxième ou de troisième ordre. Quant au stockage intégral des travaux terminologiques édités ou hors commerce, il soulève un délicat problème de droits d'auteur qui, de l'avis des juristes consultés, se trouve supprimé lorsque l'on n'extrait des ouvrages que des citations accompagnées de la mention de source. Cette considération juridico-commerciale vient renforcer l'argument de la qualité de l'informatique terminologique et celui (informatique, celui-là) du risque de pollution du fichier, sur lesquels s'est fondée au départ la décision de prétraiter la matière mise en mémoire.

Ceci dit, il reste à signaler une différence notable entre les critères de sélection retenus par la Banque de terminologie de la C.C.E. et ceux qu'a adoptés la Banque de l'Université de Montréal. Se méfiant à juste titre des lacunes de la recherche ponctuelle (ainsi désignée par opposition à la recherche thématique), la Banque de la C.C.E., où seuls les terminologues disposent de terminaux, ne consigne sur ordinateur que la terminologie de secteurs entiers et relègue à la consultation manuelle les fiches terminologiques disparates. À l'Université de Montréal, par contre, où l'on vise à doter de terminaux les traducteurs eux-mêmes, l'emmagasinage de vocabulaires entiers n'est certes pas exclu, loin de là, mais les fiches isolées qu'on juge valables sont versées elles aussi au fichier automatique, au moins provisoirement, parce qu'elles concernent assez souvent des termes difficiles ou très récents, presque toujours introuvables dans les dictionnaires et par conséquent susceptibles d'être très demandés par les usagers.

\section{L'ABONNEMENT}

Le contrat d'abonnement de Bell Canada stipule en outre que l'abonné a accès au fichier Termium durant les heures d'exploitation du Centre de calcul de l'Université, c'est-à-dire de 9 à $22 \mathrm{~h}$, sept jours par semaine, le dépannage machine étant toutefois exclu pendant les week-ends et les jours fériés. Quant au service d'appoint, en cas d'interrogation infructueuse ou non satisfaisante du fichier automatique, il est assuré durant les heures d'ouverture de la Banque, soit de 9 à $17 \mathrm{~h}$, du lundi au vendredi inclusivement, sauf les jours fériés.

À cela s'est ajoutée, par entente verbale, la mise en mémoire de trois ouvrages terminologiques anglais-français hors commerce, en usage chez le client, à savoir : le Lexique du téléphone - 1943 fiches (sans définitions), le Lexique général du commercial - 1500 fíches (sans définitions), l'Économique de l'ingénierie 360 fiches (avec définitions anglaises), soit au total 3803 fiches. Cette mise en mémoire a été réalisée sans frais pour l'abonné, puisqu'elle contribuait en même temps à l'enrichissement du capital de la Banque. 
Pour l'usager, le prix de revient du service comprend des frais fixes et des frais variables. Les premiers sont constitués d'abord par le prix de l'abonnement proprement dit à la Banque, au tarif annuel minimum de $\$ 3000$, auquel s'ajoutent les dépenses occasionnées au titre des télécommunications : soit, le cas échéant, l'obtention d'une ligne téléphonique et la location d'un terminal. Quant aux frais variables, ce sont ceux qui découlent de l'emploi des moyens informatiques ; ils sont fonction à la fois du temps de connexion (durée totale de la communication) et du temps machine (durée d'utilisation stricte de l'ordinateur). On peut estimer que l'ensemble des coûts autres que le prix de l'abonnement représente environ de 25 à $100 \%$ de celui-ci, variant en l'occurrence de $\$ 750$ à $\$ 3000$, ce qui fait en tout quelque $\$ 3750$ à $\$ 6000$ par année. C'est dire qu'une telle dépense ne se justifierait guère pour une consommation peu élevée; les petits usagers auraient donc avantage à se grouper, selon des modalités qui restent à déterminer.

Il y aurait intérêt à signaler ici un autre service offert par la Banque à tous les clients sans distinction, abonnés ou non : les index terminologiques. Il s'agit de listes dressées automatiquement à partir des données qui se trouvent dans la mémoire et réalisées sur commande à la mesure exacte des besoins de l'usager. Elles prennent généralement la forme de nomenclatures établies par ordre alphabétique anglais ou français, voire anglais et français à la fois, et groupant tous les termes disponibles qui appartiennent aux domaines sélectionnés par le client. On y trouve en regard de chacun, son ou ses synonyme(s) éventuel(s), sa ou ses traduction(s) dans l'autre langue et, au choix, différentes indications complémentaires telles que les sources bibliographiques et les domaines d'emploi en code, etc. Le délai d'obtention de ces index varie de 24 à $48 \mathrm{~h}$. Leur coût, qui n'est pas compris dans le prix de l'abonnement, s'élève à moins de 1,5 cent le couple de termes et, si l'on désire avoir aussi les contextes de manière à former un quasivocabulaire, il peut aller jusqu'à 5 cents la fiche, le prix du papier étant toujours calculé à part.

\section{PRÉPARATION DE L'EXPÉRIENCE}

Bien entendu, l'expérience a été précédée de quelques rencontres préparatoires, tant à l'échelon de la direction qu'à celui des cadres, et deux réunions mixtes ont été tenues par la suite afin d'en évaluer le déroulement et les résultats. Les préparatifs communs ont consisté en premier lieu dans l'élaboration du calendrier de l'opération. La durée de l'expérience ayant été fixée initialement à six mois, le programme a été agencé de façon à mettre à l'essai différentes formes de consultation, en faisant varier les deux paramètres suivants : le mode d'interrogation et l'ordre de priorité de la consultation de Termium par rapport à la documentation du client.

Ainsi, il a été décidé que, pendant le $1^{\mathrm{er}}$ et le $6^{\mathrm{e}}$ mois, $1^{\prime}$ interrogation se ferait indirectement, par l'intermédiaire d'un commis, mais que, durant les quatre autres mois, traducteurs et terminologues dactylographieraient eux-mêmes leurs demandes sur le téléimprimeur. En fait, cette disposition a été plus ou moins respectée, car on s'est vite aperçu, à l'usage, qu'il vaut mieux laisser à chacun la latitude soit de passer par le préposé (par exemple, afin d'éviter la perte de temps inhérente à une 
demande comprenant de nombreux termes), soit de faire fonctionner lui-même le terminal (notamment pour connaître tout de suite la réponse et, le cas échéant, dialoguer avec le fichier).

Il a également été convenu que, durant les deux premiers mois de l'expérience, l'abonné consulterait d'abord sa documentation interne, y compris son fichier terminologique collectif à accès manuel, Termium ne devant être interrogé qu'en cas d'échec de cette première démarche. Par contre, c'est le processus inverse qui serait suivi au cours des quatre derniers mois. Dictée par le simple bon sens, cette décision visait à faciliter les débuts du service d'appoint offert par la Banque, en lui épargnant un volume de demandes initial trop considérable.

La seconde disposition à prendre pour parachever les préparatifs communs a été l'établissement d'une échelle de délais de réponse à respecter relativement aux demandes S.V.P. L'échelle adoptée comporte quatre degrés: $1^{\text {er }}$, réponse immédiate; $2^{\mathrm{e}}$, réponse dans une heure ; $3^{\mathrm{e}}$, réponse dans $24 \mathrm{~h} ; 4^{\mathrm{e}}$, réponse dès que possible.

La Banque a fourni au client un guide général d'interrogation automatique, des exemplaires de sa classification systématique des domaines et, pour le personnel utilisateur, de nouveaux codes individuels établis conformément au système d'identification des auteurs de fiches Termium. De leur côté, les Services linguistiques de Bell Canada ont dû adapter le guide général d'interrogation aux caractéristiques propres du modèle de terminal choisi par les services techniques de l'entreprise, et ils ont mis au point des imprimés de contrôle destinés à l'appréciation statistique du nombre et de la qualité des réponses. À propos de téléimprimeur, le programme prévoyait la mise à l'essai successive de différents modèles d'appareils dont Bell Canada voulait connaître le rendement dans le domaine relativement nouveau des applications linguistiques. Malheureusement, les appareils avec minuscules et accents n'ayant pu être prêts à temps, l'expérience a dû se faire entièrement à l'aide d'un modèle courant qui se prête moins bien aux besoins de l'automatisation terminologique.

Pour faciliter la mise en route de l'expérience, la Banque a mis à la disposition de l'abonné, pendant quelques jours, les services d'un moniteur afin d'initier son personnel à l'interrogation du fíchier. Cette précaution n'était pas inutile, car, en plus de se familiariser avec l'aspect purement mécanique du processus ${ }^{4}$, l'interrogateur doit absolument apprendre certaines recettes qui permettent d'obvier aux inévitables lacunes de la mise en mémoire et d'obtenir ainsi un rendement maximum du capital déjà stocké en ordinateur, faute de quoi une bonne partie des ressources terminologiques de la Banque risquent d'être gravement sous-utilisées. Quelques exemples n'étant pas de trop pour illustrer ce fait, nous avons extrait des archives du service S.V.P. divers cas d'interrogation infructueuse et nous donnons entre parenthèses, à la suite de chaque terme demandé, le terme pertinent qui se trouvait en mémoire et auquel le demandeur aurait pu, avec un sens plus poussé de l'asso-

4. Rappelons ici l'aventure un peu cocasse survenue à un interrogateur novice qui avait omis de spécifier le domaine d'emploi du terme demandé. L'imprimante dévida des mètres de réponse, notre apprenti sorcier n'ayant pu "fermer le robinet", parce qu'il ne se souvenait pas de la manœuvre à exécuter et qu'il n'eut évidemment pas le temps de trouver la consigne pertinente dans le guide d'utilisation. L'automate infernal s'arrêta done de lui-même quand il fut au bout de son rouleau. 
ciation, avoir accès directement: subhead (subheading), load arm (loading arm), file cabinet (filing cabinet), rate of wages (wage rate), payback time (payback period), market research firm (market research). Le relevé systématique de ces ratages devrait permettre de dégager, avec le temps, une typologie exhaustive des omissions et maladresses contre lesquelles il faut mettre en garde aussi bien les simples interrogateurs que les terminologues chargés d'établir les déclarations d'entrée qui donnent accès à chaque fiche en mémoire.

\section{INTERROGATION DU FICHIER AUTOMATIQUE}

Le nombre de termes demandés au fichier Termium a été de 994 , soit une moyenne de 8 par jour ouvrable. Comme l'effectif du client est de 23 personnes, cela représente une demande par jour pour 3 usagers, chiffre légèrement inférieur aux résultats obtenus par la Banque de terminologie de la C.C.E., où le maximum est d'une demande par jour pour 2 usagers. On doit tenir compte ici de plusieurs facteurs. Il y a d'abord la force d'inertie due aux habitudes acquises, à laquelle se heurte nécessairement, au début, toute nouvelle méthode de travail fondée sur un rccours accru à la machine, ce désavantage n'étant pas nécessairement compensé par l'attrait d'un instrument de travail un peu futuriste. Il faut ajouter à cela le fait que, durant les deux premiers mois de l'expérience, les services de la Banque devaient, par convention, se limiter à un rôle d'appoint à l'égard du fichier de la Bell Canada. Enfin, on ne doit pas sous-estimer non plus l'entrave indéniable que constitue pour l'usager l'obligation de remplir, pour chaque demande faite pendant la durée de l'expérience, un imprimé administratif, formalité pourtant indispensable à l'étude future des résultats. Mais, de l'avis général, le plus grand obstacle a sûrement été l'effet psychologique très défavorable des interruptions de service causées par les pannes machine de la première heure. C'est du reste pour surmonter en partie certains de ces inconvénients que le client accepta volontiers d'appliquer, pendant les deux derniers mois de l'expérience, un programme individualisé d'interrogation intensive, suivant lequel chaque semaine deux membres de son personnel étaient particulièrement chargés, à tour de rôle, d'interroger à fond la Banque sur les difficultés terminologiques rencontrées dans leur travail.

Les 994 termes demandés l'ont été en quelque 177 séances d'interrogation, ce qui donne une moyenne de 1,4 séance par jour ouvrable, d'où il ressort que l'abonné laissait probablement son terminal ouvert toute la journée (pratique recommandée par la Banque dans le cas d'une utilisation intensive) ou qu'il a peut-être cherché à grouper le plus possible les demandes dans les cas de nonurgence. Ce groupement représente certes une décennie d'efforts pour le préposé, mais il pourrait se réaliser au détriment de la célérité et sans réduire pour autant les frais de téléinformatique. Les interrogations ont apporté au client environ 299 réponses automatiques, donc immédiates, soit un taux de succès de $30 \%$, c'est-àdire presque le double du rendement prévu par les estimations empiriques pour un fichier de la taille de Termium. Enfin, le nombre moyen de fiches obtenues pour chaque problème posé s'établit à 3 . Il y aurait peut-être lieu d'ouvrir ici une parenthèse pour mentionner que l'épuration systématique du fichier n'avait pas encore été entreprise à ce moment-là. Aussi l'interrogateur pouvait-il recevoir, pour un même problème, plusieurs fiches comportant des solutions identiques. Dans la 
plupart des cas, il s'agissait de fiches mises en mémoire à des dates différentes, pendant les premières années d'existence de la Banque, à l'époque héroïque où ses terminologues ne disposaient pas encore de terminaux. Comme les vérifications préalables en vue d'empêcher les doubles emplois devaient alors se faire obligatoirement au moyen d'un fichier-sosie manuel, accessible par l'anglais seulement et dépourvu de renvois pour les synonymes, on imagine assez bien tous les aléas qu'entraînait une méthode de travail aussi primitive.

\section{LE SERVICE S.V.P.}

Les 695 demandes restées sans réponse immédiate satisfaisante, à la suite de l'interrogation automatique, ont toutes été transmises au service S.V.P., grâce au service d'appoint prévu par l'abonnement, au moyen de messages spéciaux communiqués par terminal. Cette activité représente, pour les terminologues de la Banque, une moyenne de 5,5 recherches manuelles par jour ouvrable, toujours pour un abonné dont l'effectif est de vingt-trois personnes. Du point de vue de la langue, ces demandes se répartissent ainsi : $92,5 \%$ de l'anglais au français et $7,5 \%$ du français à l'anglais. En outre, $81 \%$ étaient pourvues d'un contexte, en langue de départ ou en langue d'arrivée, la plupart des autres étant accompagnées d'une simple mention de domaine, exprimée quelquefois selon le code de la Banque, mais le plus souvent rédigée en langage clair. La ventilation des demandes par domaines, donnée ci-dessous, permet de dégager un profil approximatif du client. Approximatif, parce que ce sont là des données de seconde étape, le vrai profil ne pouvant guère être fourni que par l'interrogation automatique, dont les chiffres par domaine, qui ne sont pas fournis par le logiciel actuel, ne pourraient provenir que d'un dépouillement manuel des imprimés de contrôle établis par l'abonné. Néanmoins, un rapprochement partiel - car les données ne sont pas toutes comparables - entre ce profil de client et l'état du capital terminologique de la Banque (1 ${ }^{\text {r }}$ tableau) permet déjà d'observer que les domaines caractérisés par un pourcentage à la fois très bas dans le premier tableau et très élevé dans le second (par exemple, le Droit) semblent être de ceux où il faudrait consentir un effort de recherches particulièrement important.

\section{RÉPARTITION PAR DOMAINES DES DEMANDES S.V.P.}

(Classement par orơre de fréquence décroissant)

\begin{tabular}{lc}
\multicolumn{1}{c}{ Domaines } & Pourcentages \\
Administration et gestion & 21 \\
Droit & 16,5 \\
Économie & 14,5 \\
Cybernétique & 11 \\
Télécommunications & 6,5 \\
Électricité & 4 \\
Imprimerie & 3 \\
Mécanique & 3 \\
Construction & 2,5 \\
Automobile & 2 \\
Manutention & 2 \\
Autres 5 & 14
\end{tabular}

5. Audiovisuel, crochet, démographie, dessin, diplômes, électroménager, emballage, forage, ingénierie, journalisme, marine, mathématiques, médecine, métallurgie, meuble, papier, pédagogie, pétrole, photographie, postes, sociologie, sport, statistique, transport, urbanisme. 
On peut sans inconvénient regrouper en deux catégories les quatre cotes de délais attribuées aux demandes S.V.P. : on obtient ainsi d'une part les demandes à court terme, c'est-à-dire les degrés 1 (réponse immédiate) et 2 (réponse dans une heure), et d'autre part les demandes à moyen terme, soit les degrés 3 (réponse dans $24 \mathrm{~h}$ ) et 4 (plus de $24 \mathrm{~h}$ ). Les premières comptent pour $72 \%$ et les secondes pour $28 \%$. Il n'est nul besoin de préciser que ces données ont une incidence très forte sur la valeur des réponses, car on pourrait poser en principe que la qualité des solutions est généralement proportionnelle au temps qu'on peut consacrer à la recherche.

Dans $2 \%$ des cas, les recherches effectuées se sont soldées par un échec, soit à cause de l'insuffisance des données fournies par le demandeur ${ }^{6}$, soit en raison des lacunes documentaires de la Banque ${ }^{7}$. Quant aux recherches fructueuses, les pourcentages se répartissent de la sorte : solutions attestées intégralement dans des sources dignes de foi $(46,5 \%)$, solutions attestées partiellement dans des ouvrages en langue originale ou relevées dans de simples dictionnaires de traduction $(41,5 \%)$, solutions non attestées $(10 \%)$. Les recherches effectuées ont abouti à la création de 718 fiches $(79,5 \%$ à deux contextes, $19,5 \%$ à un seul contexte et $1 \%$ sans contexte) portant sur les termes mêmes qui avaient été demandés. Elles ont aussi donné lieu à l'établissement de 68 fiches à deux contextes, mais portant sur des termes connexes à ceux qui faisaient l'objet de la demande, ce qui donne en tout 786 fiches nouvelles.

Les réponses S.V.P., dont l'envoi initial se fait aussi par terminal, sont ensuite mises en mémoire le plus tôt possible, c'est-à-dire dans un délai qui en principe ne devrait pas dépasser une semaine, afin que l'abonné puisse y avoir de nouveau accès à volonté. Cette dernière étape doit toutefois être précédée d'une révision, qui a nécessité en l'occurrence quelque 115 corrections de toute sorte ${ }^{\mathrm{s}}$, dont la teneur a été communiquée au client au fur et à mesure du processus. Le taux de corrections différées atteint donc $17 \%$ des demandes S.V.P., ce qui n'est pas exorbitant si l'on tient compte du climat de hâte dans lequel les terminologues doivent travailler pour arriver à respecter les échéances souvent très courtes qui leur sont imposées.

Pour ce qui est de la durée des recherches, elle totalise environ 814 heures auxquelles s'ajoute le temps consacré à la révision et que l'on pourrait, faute de statistique, évaluer sommairement à un quart d'heure par fiche, en moyenne, ou 174 heures pour 695 termes, ce qui donne un grand total de 988 heures de travail. Si l'on compare cette donnée à l'effectif du client ( 23 personnes) et aussi à l'horaire de travail semestriel d'un terminologue, qui s'établit à 690 heures, on peut en conclure que le nombre de personnes desservies par un terminologue est de 16 , pour un volume de demandes ne dépassant pas le chiffre indiqué plus haut. D'autre part, la durée moyenne de la recherche proprement dite se chiffre à $1 \mathrm{~h} 15$ par terme demandé, mais cette valeur se trouve ramenée à $1 \mathrm{~h}$ par fiche créée si

6. Exemple: (mécanique) sheave leg, tilt and angle brace, uni-dyne bearing; (téléinformatique) $n$ key roll-over, pilot blocking.

7. Exemple: (imprimerie) crash imprint; (mécanique) to pick up the load; (télécommunications) crossover trunk call; (téléinformatique) parity keyboard, scan printout.

8. Exemple : (télécommunications) dummy plug, loop extender, pulse-link repeater. 
l'on tient compte de l'établissement de fiches incidentes, c'est-à-dire portant sur des termes connexes au problème de départ.

À un certain moment, les terminologues de Bell Canada ont regroupé, dans des demandes échelonnées sur quelques semaines, plus de 150 termes du secteur de l'immobilier ${ }^{9}$, de manière à former en définitive un véritable micro-vocabulaire. Ils ont aussi cédé à la Banque une trentaine de fiches originales dont la plupart sont consacrées à des termes de télécommunications.

Un peu plus tard, ils ont aussi obtenu, par correspondance, l'avis des terminologues de la Banque sur une trentaine de fiches originales portant pour la plupart sur des problèmes de télécommunications.

Une autre fois, la Banque a reçu par terminal une demande de documentation française sur la capitalisation et elle y a accédé au mieux de ses moyens, par la poste. De plus, tout au long de l'expérience, les interrogateurs se sont généralement fait un devoir de signaler à la Banque les réponses qui leur paraissaient fautives ou vraiment insatisfaisantes.

Ces exemples méritent d'être cités, car ils illustrent bien l'esprit de collaboration qui régnait entre les deux équipes. Ils suppléent au langage des chiffres, impuissant par nature à exprimer des réalités comme l'humour qui se manifeste dans certains messages ${ }^{10}$.

Pour terminer, venons-en à l'ultime question, celle que tout le monde se pose, à côté de laquelle toutes les autres n'ont, au fond, pas beaucoup d'importance : et les usagers, eux, qu'en pensent-ils ? Car, en définitive, si la Banque de terminologie répond plus ou moins à leurs attentes, son utilité s'en trouve diminuée et ses promoteurs devront tôt ou tard s'interroger sérieusement sur son avenir. D'un sondage maison effectué auprès d'eux, il ressort que leur degré de satisfaction se chiffrerait entre 65 et $70 \%$, ce qui est un bon commencement. Pourtant, étant donné l'enjeu de la question, il est à souhaiter qu'une enquête plus approfondie soit menée par un organisme impartial, sur des bases scientifiques, afin d'amener les utilisateurs à livrer le fond de leur pensée sur l'expérience à laquelle ils ont pris part. Parions que seraient ainsi mis en lumière des aspects de la question qui n'ont été jusqu'ici qu'effleurés, sinon tout à fait négligés ${ }^{11}$.

Bruno Couture

9. Exemple : abstract index, exclusive listing, land registration, metes and bounds, Torrens system.

10. Un bon échantillon de celui-ci nous est donné par la personne qui, ayant demandé le terme fish wire le 1er avril (jour des traditionnels poissons), s'est crue obligée d'ajouter que sa demande était des plus sérieuses. Et il y a même eu, à l'occasion d'une panne de l'ordinateur (de modèle Cyber), ce jeu de mots fondé sur un juron local bien connu : "Le Cibouère ne fonctionne pas ".

11. Les personnes qui voudraient de plus amples informations sur la Banque peuvent en obtenir en écrivant à l'adresse suivante : Banque de terminologie, Université de Montréal, Beîte postale 6128, Succursale "A ", Montréal, Qué., H3C 3J7. 